\title{
Pinzamiento femoroacetabular: Conceptos básicos en una nueva causa de dolor inguinal
}

\author{
RODRIGO MARDONES P. ${ }^{1,2}$, VÍCTOR BARRIENTOS C. ${ }^{1}$, \\ FERNANDO NEMTALA U. ${ }^{1}$, ALEXANDER TOMIC $^{1}$, MATÍAS SALINEROS U. ${ }^{1}$
}

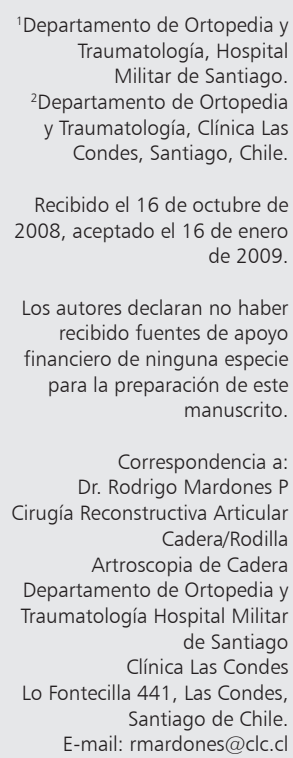

\section{Femoroacetabular impingement as a cause of inguinal pain}

Femoroacetabular impingement is an anatomical disturbance of the hip, caused by a deformity of the acetabulum, femur or both that causes an abnormal contact between both structures during certain movements. Its prevalence is 10 to $15 \%$ and causes chronic inguinal pain. It can be confused with several other causes of inguinal pain such as hernias, facet syndromes, a renal colic, etc. Patients with this condition are usually young individuals with inguinal pain that may appear after a minor trauma. During examination, pain may be elicited by internal rotation and abduction movements of hip, flexed in $90^{\circ}$. Plain hip $X$ ray is the most commonly used diagnostic method. Non steroidal anti inflammatory drugs and physical therapy can be used to alleviate pain, but the definitive treatment is surgical

(Rev Med Chile 2010; 138: 102-108).

Key words: Anti-inflammatory agents, Non-steroidal; Hip; Osteoarthritis, Hip.
$\mathrm{E}$ 1 dolor inguinal es una causa frecuente de consulta, tanto a nivel de medicina general como de especialidades. Se debe tener en cuenta que en la región inguinal existen varios sistemas "sobrepuestos", como son el sistema músculo esquelético, génito urinario, gastrointestinal y distintas estructuras neurovasculares; cualquiera de estas pueden ser fuente de dolor inguinal, por esto se debe estar familiarizado con el estudio y eventual tratamiento de distintas enfermedades. En este contexto creemos necesario actualizar y difundir dentro de la comunidad médica algunos conceptos en relación a una patología en particular como causa de dolor inguinal, el síndrome de pinzamiento femoroacetabular (PFA), ya que con una prevalencia entre el 10 y $15 \%{ }^{1}$, aún es poco reconocida como causa de dolor inguinal, tanto entre traumatólogos, médicos generales y otras especialidades médicas (neurocirugía, cirugía general, ginecología, urología, medicina interna), de tal manera que muchos pacientes son diagnosticados y tratados por distintas enfermedades que se confunden clínicamente con este síndrome (endometriosis, cólico renal, hernia inguinal, cruralgias, síndrome facetario, síndrome piriforme, fracturas por estrés, desgarros musculares, esguinces, tendinitis, bursitis, etc) ${ }^{2}$. Los síntomas persistentes y manejo inadecuado llevan al paciente a consultar distintos especialistas y subespecialistas, siendo sometidos incluso a variados procedimientos quirúrgicos (laparoscopias, laparotomías, artroscopias de rodilla, reparación de hernias inguinales, bloqueos facetarios, discectomías, infiltraciones articulares y tendineas, etc) ${ }^{2,3}$, muchas veces $\sin$ 
llegar al diagnóstico correcto y su consiguiente tratamiento. El avance en los últimos años sobre todo en el estudio de imágenes ha permitido identificar anormalidades anatómicas que hasta ahora habían sido subdiagnosticadas o desconocidas, como las encontradas en el síndrome de pinzamiento femoroacetabular. Además, con la utilización de la artroresonancia se ha logrado mejorar la sensibilidad en el diagnóstico de lesiones asociadas de cartílago acetabular y labrum, que explicarían la persistencia de dolor en este tipo de pacientes. Un estudio imagenológico compatible asociado a la abolición del síntoma doloroso, con el uso de infiltración intraarticular de lidocaína (test de lidocaína positivo) permite confirmar el diagnóstico. Se ha propuesto que el PFA acelera el proceso degenerativo articular y desencadena la aparición de artrosis ${ }^{3}$.

\section{Tipos de Pinzamiento}

Se han descrito dos tipos de pinzamiento fémoroacetabular basado en las observaciones intraoperatorias de las lesiones condrales y de labrum en sus distintos grados.

\section{Tipo "cam" o "en leva"}

Es causado por el atrapamiento de una cabeza femoral anormal con un radio en aumento en movimiento extremo, especialmente la flexión ${ }^{3}$ (Figura 1A). La causa de base para la morfología anormal en los pellizcamientos de leva ( $\mathrm{cam}$ ) no está claramente esclarecida. Algunas causas probables son alteraciones del desarrollo, siendo la más probable la epifisiolisis mínima subclínica con la consecuente disminución del offset cabeza-

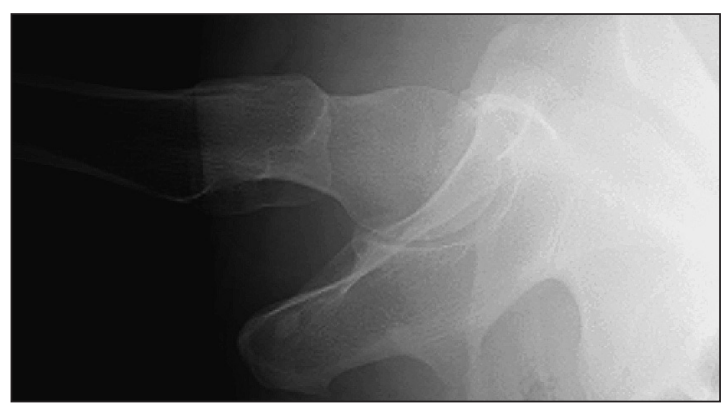

Figura 1A. Pinzamiento tipo "cam": prominencia ósea en unión cabeza-cuello femoral. cuello anterior. Esta disminución del offset genera un contacto anormal entre la unión cabeza cuello y el reborde acetabular ${ }^{4}$. Las fuerzas de fricción resultantes llevan a una abrasión de afuera a adentro del cartílago acetabular o avulsión del labrum y hueso subcondral en un área ántero superior relativamente constante. La lesión condral lleva al desgarro o desinserción del labrum no involucrado inicialmente. Las fracturas de cuello femoral también pueden generar una situación similar al consolidar con retroversión cervical ${ }^{5,6}$. El pellizcamiento "de leva", con una frecuencia cercana al $6 \%$, es más común en individuos atléticos jóvenes de sexo masculino ${ }^{7}$.

\section{Tipo "pincer" o "en tenaza"}

Es el resultado de un contacto lineal entre el reborde acetabular y la unión cabeza-cuello femoral. La cabeza puede ser morfológicamente normal, y el pellizcamiento producto de una anormalidad acetabular, generalmente sobrecobertura (coxa profunda/protrusión acetabular) o local (retroversión acetabular $)^{7}$ (Figura 2A). La primera estructura en fallar en estos casos es el labrum acetabular. El impacto continuo lleva a degeneración del labrum, y se producen gangliones u osificación del reborde llevando a una profundización del acetábulo empeorando la sobre cobertura. El efecto de estribo, que generalmente es anterior, lleva a una palanca sobre el fémur presionándose la cabeza contra el acetábulo en la zona póstero inferior. Las lesiones condrales en el pellizcamiento tipo "pincer" usualmente son limitadas a un área de reborde pequeña siendo más benignas ${ }^{8}$. El pellizcamiento "en tenaza" tiene una frecuencia de $9 \%$ y es más común en mujeres de edad media con algún grado de actividad deportiva ${ }^{3}$.

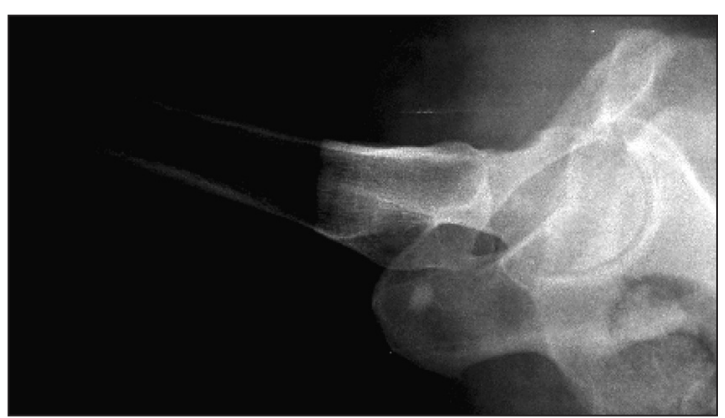

Figura 1B. Resultado de corrección de deformidad mediante técnica artroscópica. 


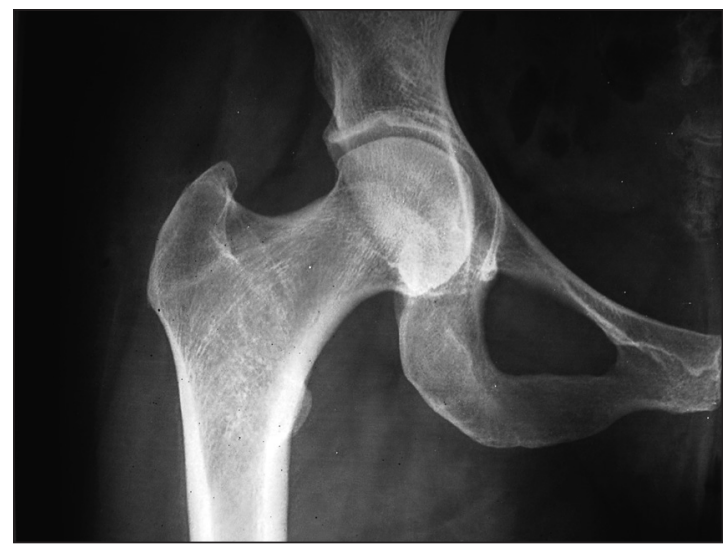

Figura 2A. Pinzamiento tipo "Pincer": protrusio acetabular, cabeza femoral sobrepasa línea ilioisquiática, existe cobertura $100 \%$ de cabeza femoral.

\section{Pellizcamiento mixto}

El pinzamiento mixto es el más frecuente encontrándose hasta en $86 \%$ de los casos y combina en distinto grado los dos tipos descritos previamente ${ }^{3}$. Aparentemente se trataría inicialmente de un pinzamiento tipo "pincer o tenaza" que posteriormente desarrollaría la aparición del bump anterolateral a nivel de la unión cabeza cuello, produciéndose un pinzamiento tipo "cam o leva" secundario, con lo que se obtiene la presencia simultánea de ambos tipos de pinzamiento.

\section{Diagnóstico}

Se debe tener en cuenta que en la región inguinal existen varias fuentes potenciales de dolor, debido a la presencia de varios sistemas "sobrepuestos", como son el sistema músculo esquelético, génito urinario, gastrointestinal y distintas estructuras neurovasculares. Como se describió previamente, el pinzamiento femoroacetabular debe ser considerado dentro de un amplio número de diagnósticos diferenciales planteables ante un dolor inguinal tanto en pacientes de sexo masculino como femenino (Tabla 1).

\section{Clínica}

Los pacientes con síndrome de pinzamiento femoroacetabular habitualmente son jóvenes, entre 20 y 40 años de edad. La prevalencia estimada de esta patología es de 10 a $15 \%{ }^{1}$.

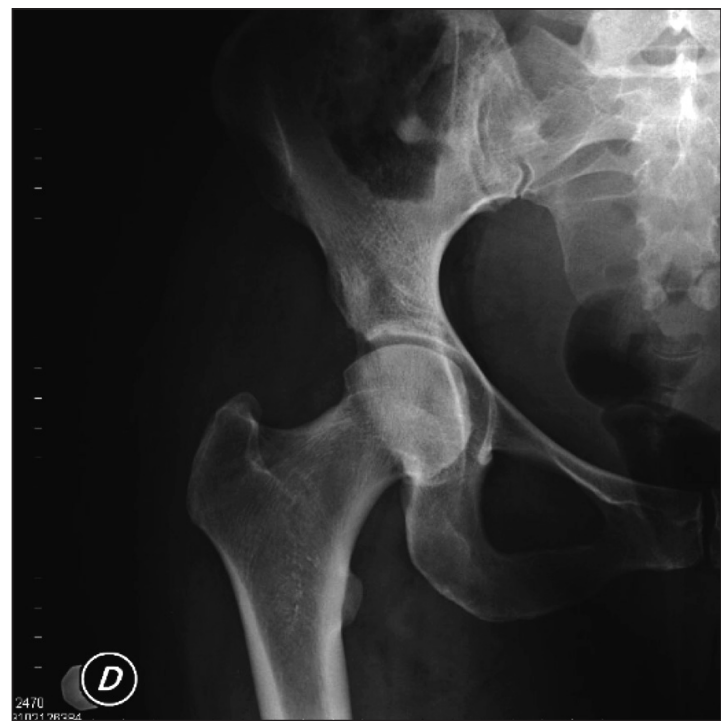

Figura 2B. Resultado de resección de sobrecobertura mediante técnica artroscópica, se obtiene cobertura de 80\% de cabeza femoral, logrando corregir pinzamiento articular.

\section{Tabla 1. Diagnósticos diferenciales dolor inguinal en el adulto}
1. Endometriosis
2. Cólico renal
3. Varicocele
4. Epididimitis
5. Hernia inguinal
6. Linfangitis
7. Hernia del deportista
8. Pubalgia del deportista
9. Cruralgías
10. Síndrome facetario
11. Síndrome piriforme, meralgia preestética, atrapa- miento nervio pudendo u obturador
12. Fracturas por estrés fémur proximal o ramas ilio e isquiopúbicas
13. Desgarros musculares sartorio, aductores, recto femoral, iliopsoas
14. Esguinces
15. Tendinitis glútea, rotadores externos de cadera, psoas iliaco
16. Bursitis trocánter mayor o menor, iliopsoas, tube- rosidad isquiática
17. Necrosis avascular cabeza femoral
18. Coxartrosis 
El síntoma cardinal de esta patología es el dolor que habitualmente se ubica en la región inguinal de la cadera afectada, este puede ser de aparición brusca en relación a algún traumatismo, muchas veces de escasa energía o de aparición insidiosa sin mecanismo causal evidente. Se describe el "C sign" donde el paciente indica una zona dolorosa poco definida, con el pulgar abducido sobre la región trocantérica y glútea, y el índice hacia la región inguinal (Figura 3). Sin embargo, muchas veces puede existir dolor referido a otras localizaciones como la rodilla.

Con frecuencia estos pacientes perciben una limitación del rango de movilidad articular de la

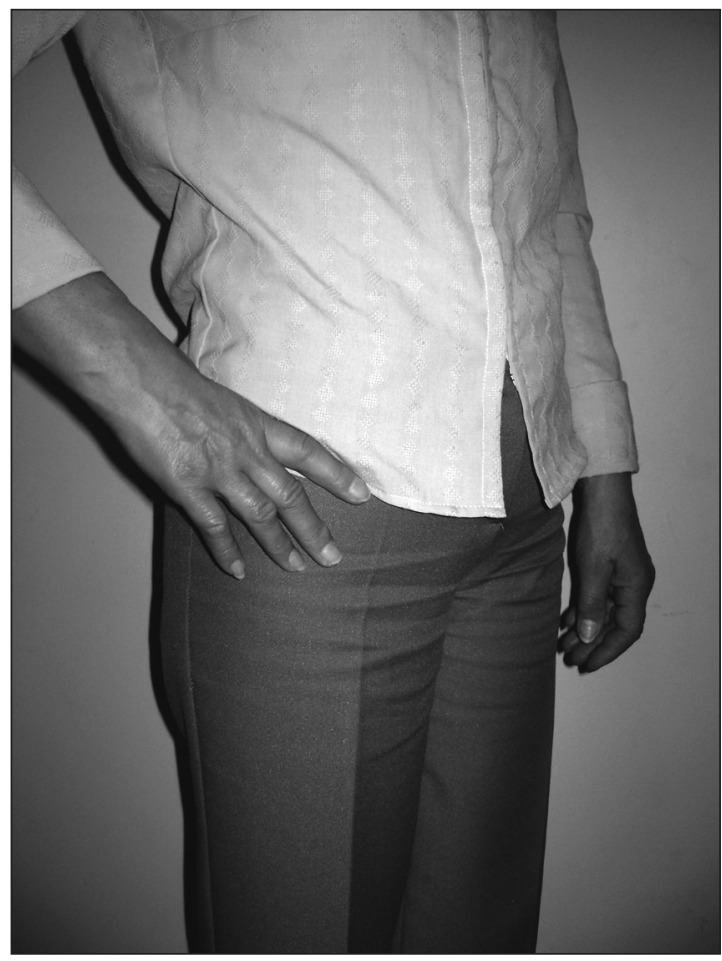

Figura 3. Muestra imagen con "C sign" o "Signo de la C". cadera mucho antes de la aparición de sintomatología dolorosa.

El dolor suele desencadenarse con los movimientos de rotación interna y aducción con la cadera flectada a $90^{\circ}$, maniobra conocida como test de pellizcamiento ${ }^{3}$.

Los pacientes refieren dolor asociado a actividades de la vida diaria como subir escaleras, bipedestar después de sedestación prolongada, moverse en la cama, subir y bajarse del automóvil, así como también durante o después de realizar actividades deportivas, durante estas actividades se efectúan los movimientos que reproducen el dolor.

En el pinzamiento anterosuperior, que es el más frecuente, generalmente existe limitación del rango de movilidad de flexión y rotación interna.

En casos de rotura de labrum los pacientes refieren con frecuencia una sensación de chasquido o bloqueo doloroso del rango de movilidad articular.

\section{Examen Físico}

Al examen de la marcha el paciente puede presentar desde una claudicación severa con la mano apoyada en la región dolorosa ("C sign") hasta una marcha indolora.

Con el paciente en posición supina, se examinan los rangos articulares de la cadera sana y enferma posteriormente, se sugiere consignarlos en una tabla (flexión, extensión, rotación interna, rotación externa, aducción, abducción, rotación interna en $90^{\circ}$, test de pellizcamiento y FABER) (Tabla 2).

Habitualmente presentan disminución de la rotación interna de cadera, test de pellizcamiento positivo (se reproduce el dolor al llevar la cadera en flexión de $90^{\circ}$, efectuando aducción y rotación interna). Frecuentemente se observa un FABER aumentado con tope articular (cadera en flexión, abducción y rotación externa) o doloroso?.

Tabla 2. Valores normales de rango articular de caderas. FABER con 0 ó 1 puño corresponde a distancia de la rodilla hasta camilla

\begin{tabular}{|cccccccccc|}
\hline Flexión & $\begin{array}{c}\text { Exten- } \\
\text { sión }\end{array}$ & $\begin{array}{c}\text { Rotación } \\
\text { Interna }\end{array}$ & $\begin{array}{c}\text { Rot. } \\
\text { Externa }\end{array}$ & Abducción & Adducción & $\begin{array}{c}\text { Rot. } \\
\text { Interna } \\
\mathbf{9 0 ^ { \circ }}\end{array}$ & $\begin{array}{c}\text { Test } \\
\text { Pellizcamiento }\end{array}$ & FABER \\
\hline DER & $100^{\circ}$ & $0^{\circ}$ & $35^{\circ}$ & $45^{\circ}$ & $45^{\circ}$ & $20^{\circ}$ & $20^{\circ}$ & Negativo & $0-1$ puño \\
\hline IZQ & $100^{\circ}$ & $0^{\circ}$ & $35^{\circ}$ & $45^{\circ}$ & $45^{\circ}$ & $20^{\circ}$ & $20^{\circ}$ & Negativo & $0-1$ puño \\
\hline
\end{tabular}




\section{Imagenología}

El estudio complementario de imágenes es muy importante para la identificación de anormalidades anatómicas asociadas al pinzamiento, y para descartar otras patologías tanto de origen extraarticular como articulares, por ejemplo necrosis avascular, artrosis, tumores, tendinitis, bursitis y otros.

Las radiografías simples siguen siendo el método más accesible y difundido para el diagnóstico de esta patología y debe ser siempre el examen inicial. Las proyecciones recomendadas para el diagnóstico son: pelvis anteroposterior, cadera anteroposterior y cross-table (axial verdadera) de la cadera afectada.

La proyección anteroposterior de la pelvis

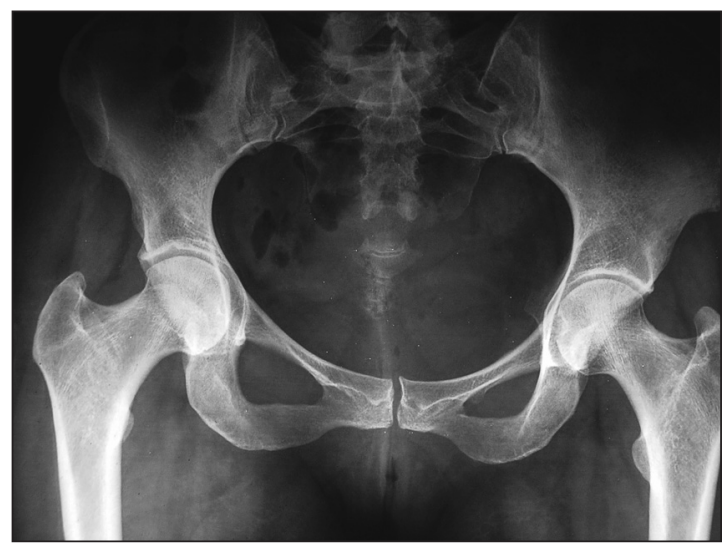

Figura 4. Protrusio acetabular bilateral retroversión acetabular cadera izquierda, destaca entrecruzamiento de pared anterior y posterior.

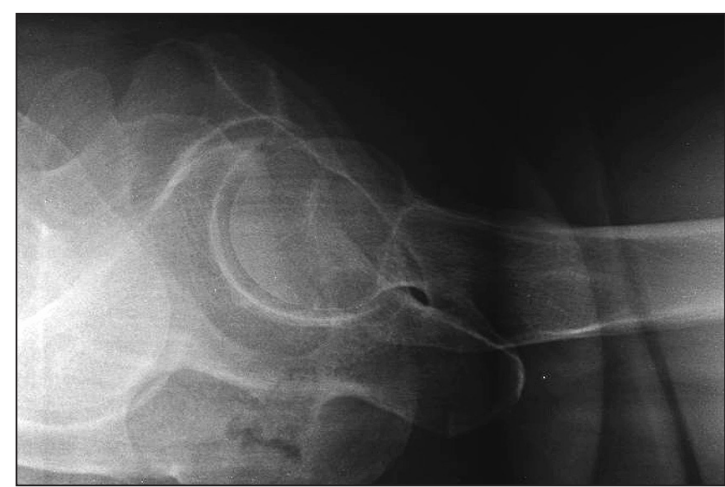

Figura 5. Rx cross table, ángulo alfa normal, offset anterior conservado. permite identificar casos de displasia acetabular, retroversión acetabular y grado de cobertura acetabular (Figura 4). La proyección anteroposterior de la cadera afectada permite observar con mayor detalle la unión cabeza-cuello lateral junto con las alteraciones descritas previamente.

La proyección cross table es útil para analizar la unión cabeza-cuello femoral tanto anterior como posterior. En condiciones normales la concavidad anterior de ese segmento debe ser similar a la posterior (offset) (Figura 5), la disminución de la concavidad anterior (offset anterior) debe hacer sospechar la presencia de bump anterior (Figura 6).

La resonancia magnética y la artroresonancia magnética son muy útiles para evaluar el labrum acetabular y el cartílago articular, es capaz de detectar alteraciones en la esfericidad de la cabeza femoral, cuellos femorales cortos, depresiones por herniación, u osificación del reborde, el cuello femoral puede presentar depresiones por hernias o hendiduras, que se postula, indicarían pellizcamiento $^{10}$. Así como otras estructuras blandas, intra y extra articulares (sinovitis, lesión del ligamento redondo, cuerpos libres intraarticulares, bursitis, tendinitis).

La artroresonancia, a diferencia de la resonancia magnética convencional (sin contraste intraarticular), permite una mejor evaluación de roturas del labrum, las cuales se identifican al ver medio de contraste atravesando la línea del labrum, y las lesiones condrales por acumulación de medio de contraste atravesando el cartílago o creando una interfase osteocondral subyacente (Figura 7). Los cambios fibroquísticos son prevalentes en los casos de pellizcamiento femoroacetabular y se describe

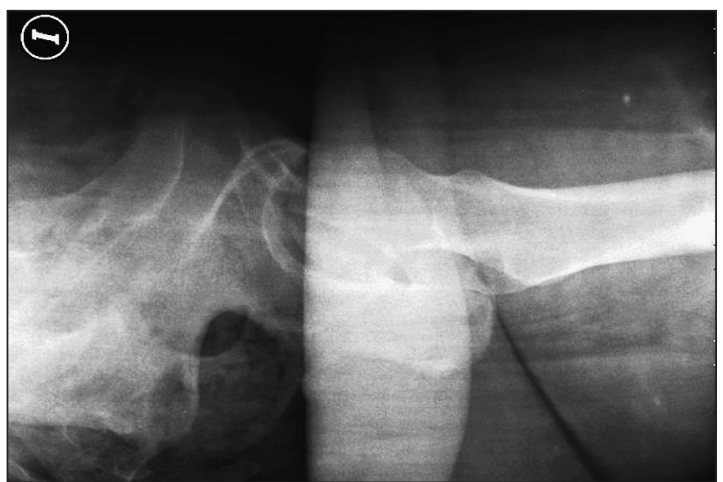

Figura 6. Rx cross table, muestra ángulo alfa alterado, offset anterior disminuido por presencia de bump. 


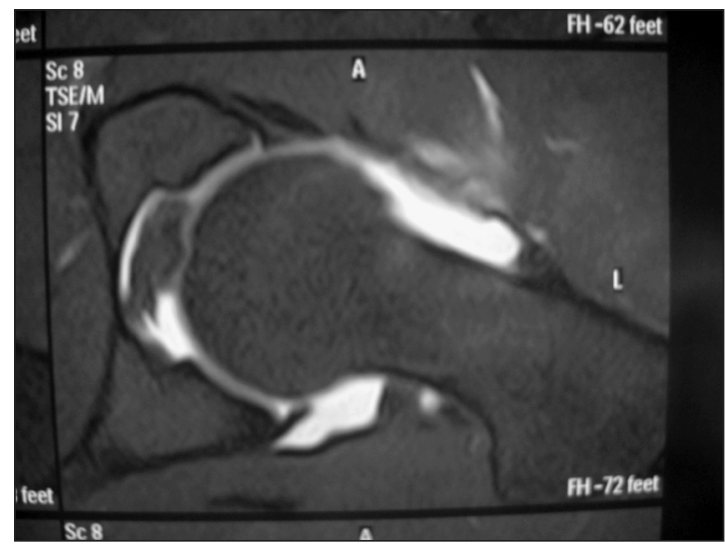

Figura 7. Artro resonancia magnética, muestra rotura labrum acetabular.

una asociación entre los quistes y daño al labrum o cartílago adyacente.

Durante la infiltración de medio de contraste intraarticular se puede además colocar lidocaína al 2\% (test de lidocaína), en presencia del anestésico local intraarticular y la abolición del dolor, junto con el estudio imagenológico compatible, se puede confirmar el diagnóstico.

En la artroresonancia magnética de cadera se describe el ángulo alfa, que es un parámetro de esfericidad de la cabeza femoral. El ángulo alfa fue descrito por Notzli et al y consiste en trazar una línea desde el centro de la cabeza femoral hasta el punto del contorno de la cabeza femoral que se encuentra a una distancia mayor al radio de la cabeza femoral. Se traza una segunda línea que corresponde al eje del cuello femoral y que atraviesa el centro de la cabeza femoral ${ }^{11}$. El ángulo alfa se forma por ambas líneas, cuanto mayor sea el ángulo, menos esférica será la cabeza femoral. Se describe para la población normal un ángulo alfa menor a $50^{\circ}$.

\section{Tratamiento}

De manera paliativa pueden prescribirse antiinflamatorios y kinesioterapia con énfasis en ejercicios de fortalecimiento de musculatura periarticular, sin embargo los resultados de este tratamiento conservador son poco predecibles.

El tratamiento definitivo del síndrome de pinzamiento femoroacetabular es quirúrgico, corrigiendo la alteración anatómica causante del contacto anormal entre el fémur y el reborde acetabular, una vez que esta ha sido identificada.

Los procedimientos consisten básicamente en tallar el reborde óseo acetabular (acetabuloplastía), eliminando la sobrecobertura (pincer) y la unión cabeza-cuello femoral (bumpectomía), evitando resecar más de $30 \%$ de ésta ${ }^{12}$, de esta forma se mejorara el offset anterior femoral. Esto puede realizarse de forma artroscópica ${ }^{13} \mathrm{o}$ abierta a través de una luxación controlada de cadera ${ }^{14,15}$. En algunos casos de retroversión acetabular severa es necesario realizar una osteotomía periacetabular reversa $^{3,14-17}$.

El labrum acetabular lesionado puede resecarse o en lo posible repararse, principalmente en gente joven, con el fin de restituir el efecto de sello de la articulación que produce esta estructura ${ }^{16,18} y$ preservar su función propioceptiva y de estabilizador articular ${ }^{19}$.

Los resultados de estos procedimientos son muy dependientes del grado de artrosis preexistente. Sin embargo, en pacientes sin artrosis o con artrosis leve los resultados son prometedores.

\section{Discusión}

El pinzamiento femoroacetabular con una prevalencia entre 10 y $15 \%{ }^{1}$, aún es una causa de dolor inguinal y de cadera poco reconocida, tanto en la comunidad traumatológica y médica general como de otras especialidades, lo que provoca que muchos pacientes sean tratados por cuadros que se confunden con este ${ }^{2}$ (Tabla 1 ). Jäger et al muestran un retraso promedio de 5,4 años desde el inicio de los síntomas y el diagnóstico ${ }^{20}$, por su parte Burnett et al presentan 21 meses de retardo en el diagnóstico ${ }^{21}$, los síntomas persistentes y manejo inadecuado llevan al paciente a consultar distintos especialistas y subespecialistas, con un promedio de 3,3 visitas previas al diagnóstico ${ }^{21}$, siendo sometidos incluso a variados procedimientos quirúrgicos $^{2,3}$, muchas veces sin llegar al diagnóstico correcto y su consiguiente tratamiento. Si a todo lo anterior se agrega que ya se ha identificado el pinzamiento femoroacetabular como una causa desencadenante de artrosis ${ }^{3}$, que en el pasado eran consideradas como primaria o idiopática, creemos fundamental el poder difundir en forma clara $y$ masiva dentro de nuestra comunidad médica y no solamente a nivel de traumatología, la existencia del síndrome de pinzamiento femoroacetabular, 
ya que es indispensable tener el conocimiento de este síndrome para poder plantearlo como hipótesis diagnóstica, solamente de esta manera se podrá entender una historia clínica sugerente, poder efectuar un examen físico orientado a descartar esta patología, junto con solicitar el estudio radiológico básico adecuado ${ }^{22,23}$, que se encuentra disponible en gran parte del país.

\section{Referencias}

1. Leunig M, Ganz R. Femoroacetabular impingement: a common cause of hip complaints leading to arthrosis. Unfallchirurg 2005; 108: 9-17.

2. Parvizi J, Leunig M, Ganz R. Femoroacetabular Impingement. J Am Acad Orthop Surg 2007; 15: 561-70.

3. Ganz R, Parvizi J, Beck M, Leunig M, Notzli H, Siebenrock KA. Femoroacetabular impingement: a cause for osteoarthritis of the hip. Clin Orthop Relat Res 2003; 417: 1-9.

4. Leunig M, Casillas MM, Hamlet M, Hersche O, Notzli $\mathrm{H}$, Slongo T, et al. Slipped capital femoral epiphysis: early mechanical damage to the acetabular cartilage by a prominent femoral metaphysis. Acta Orthop Scand 2000; 71: 370-5.

5. Kassarjian A, Yoon L, Belzile E, Connolly S, Millis M, Palmer W.Triad of MR arthrographic findings in patients with Cam type femoroacetabular impingement. Radiology 2005; 236: 588-92.

6. Siebenrock KA, Wahab KH, Werlen S, Kalhor M, Leunig M, Ganz R. Abnormal extension of the femoral head epiphysis as a cause of cam impingement. Clin Orthop Relat Res. 2004; 418: 54-60.

7. Giori NJ, Trousdale RT. Acetabular retroversion is associated with osteoarthritis of the hip. Clin Orthop Relat Res 2003; 417: 263-9.

8. Beck M, Kahlor M, Leunig M, Ganz R. Hip morphology influences the pattern of damage to the acetabular cartilage: femoroacetabular impingement as a cause of early osteoarthritisof the hip. J Bone Joint Surg Br 2005; 87: 1012-8.

9. Byrd JWT. Physical examination. In: Byrd JWT, editor. Operative hip arthroscopy. 2nd edition. New York: Springer; 2005. p. 36-50.

10. Pitt MJ, Graham AR, Shipman JH, Birkby W. Herniation pit of the femoral neck. Am J Roentgenol 1982; 138: 1115-21.

11. Notzli H, Wyss T, Stoecklin C, Schmid M, Treiber K, Hodler J. The contour of the femoral head-neck junction as a predictor for the risk of anterior impingement. J Bone Joint Surg (Br) 2002; 84B: 556-60.

12. Mardones RM, González C, Chen Q, Zobitz M, Kaufman KR, Trousdale RT. Surgical treatment of femoroacetabular impingement: evaluation of the effect of the size of the resection. J Bone Joint Surg Am 2005; 87: 273-9.

13. Mardones R, Lara J, Donndorff A, Barnes S, Stuart MJ, Glick J, Trousdale R. Surgical Correction of "Cam-Type" Femoroacetabular Impingement: A Cadaveric Comparison of Open versus Arthroscopic Debridement. Arthroscopy: The Journal of Arthroscopic and Related Surgery. Arthroscopy 2009 Feb; 25 (2): 175-82. Epub 2008 Nov 1.

14. Mardones RM, González C, Chen Q, Zobitz M, Kaufman KR, Trousdale RT. Surgical treatment of femoroacetabular impingement: evaluation of the effect of the size of the resection. Surgical technique. J Bone Joint Surg Am 2006; 88 Suppl 1 Pt 1: 84-91.

15. Ganz R, Gill TJ, Gautier E, Ganz K, Krugel L, Berlemann U. Surgical Dislocation of the adult hip: a technique with full access to femoral head and acetabulum, without the risk of avascular necrosis. J Bone Joint Surg Br 2001; 83: 1119-24.

16. Espinosa N, Beck M, Rothenfluh D, Ganz R, Leunig M. Treatment of femoroacetabular impingement: preliminary results of labral refixation. Surgical technique. J Bone Joint Surg Am 2007; 89: 36-53.

17. Siebenrock KA, Schoniger R, Ganz R. Anterior femoroacetabular impingement due to acetabular retroversion and its treatment by periacetabular osteotomy. J Bone Joint Surg Am 2003; 85: 278-86.

18. Fitzgerald RH Jr. Acetabular labrum tears: diagnosis and treatment. Clin Orthop 1995; 311: 60-8.

19. Torri M, Schenker M, Martin H, Hogoboom D, Philippon M. Neuromuscular hip biomechanics \& pathology in the athlete. Clin Sports Med 2006; 25: 179-97.

20. Jäger M, Wild A, Westhoff B, Krauspe R. Femoroacetabular impingement caused by a femoral osseous head-neck bump deformity: clinical, radiological, and experimental results. J Orthop Sci 2004; 9: 256-63.

21. Burnett RS, Della Rocca GJ, Prather H, Curry M, Maloney WJ, Clohisy JC. Clinical presentation of patients with tears of the acetabular labrum. J Bone Joint Surg Am. 2006; 88: 1448-57.

22. Maheshwari A, Malik A, Dorr LD. Impingement of the Native Hip Joint. J Bone Joint Surg Am 2007; 89: 250818.

23. Sierra R, Trousdale RT, Ganz R, Leunig M. Hip Disease in the Young, Active Patient. J Am Acad Orthop Surg 2008; 16: 689-703. 